\title{
The Virgo Stellar Stream: Extended sample
}

\author{
S. Duffau ${ }^{1}$, A. K. Vivas ${ }^{2}$, R. Zinn ${ }^{3}$, R. A. Méndez ${ }^{4} \&$ M. T. Ruiz ${ }^{4}$ \\ ${ }^{1}$ Astronomisches Rechen-Institut, Zentrum fuer Astronomie, University of Heidelberg, \\ Moenchhofstrasse 12-14, D-692120, Heidelberg, Germany \\ email: sduffau@ari.uni-heidelberg.de \\ ${ }^{2}$ Centro de Estudios de Astronomia (CIDA) \\ Apartado Postal 264, Merida 5101-A, Venezuela \\ email: akvivas@cida.ve \\ ${ }^{3}$ Astronomy Department, Yale University \\ P.O. Box 208101, New Haven, CT 06520-8101 USA \\ email: robert.zinn@yale.edu \\ ${ }^{4}$ Departamento de Astronomia, Universidad de Chile \\ Casilla 36-D, Santaigo, Chile \\ email: mtruiz@das.uchile.cl,rmendez@das.uchile.cl
}

\begin{abstract}
We have completed a spectroscopic study of the "12.4 hr clump", the second largest substructure in the Quasar Equatorial Survey Team (QUEST) catalog. First discovered as an over-density of RR Lyrae stars (Vivas et al. 2001, ApJL 554 33), the region containing the "12.4 hr clump" has generated much interest (Newberg et al. (2002), Martinez-Delgado et al. (2007), Juric et al. (2008), amongst many others). Our first spectroscopic study of this clump revealed the presence of a sharp peak in the radial velocity histogram for the candidate stars (Duffau et al. 2006). The combination of this result and metal abundance estimates for the sample was then interpreted as a signature of the presence of a stellar stream within the clump. This substructure was named the "Virgo Stellar Stream" (VSS), given its location in the direction of the Virgo Constellation, at approximately $20 \mathrm{kpc}$ from the Sun. Several other groups have studied this region and have suggested that the over-density containing the VSS could extend to larger areas of the sky (outside QUEST's observing range). We present the complete spectroscopic follow up of the clump candidates present in QUEST and the composite of the studies we performed along the same l.o.s., including data at brighter magnitudes (Vivas et al. 2008). Our study confirmed the nature of the VSS, revealed its likely extent within the QUEST survey and defined a number of its relevant properties.
\end{abstract}

Keywords. Galaxy: halo, Galaxy: structure, stars: variables: other

\section{Introduction}

The Virgo Stellar stream, a stellar stream within a large overdensity in the direction of the constellation of Virgo is the main subject of this research. This stellar stream was discovered in 2006 (Duffau et al. 2006) while performing a spectrosocpic follow-up of the densest part of the $12.4 \mathrm{hr}$ clump, as seen by QUEST. This project has been developed on the context of a large collaboration between Yale University, Universidad de Chile and the Centro de investigaciones de Astronomia in Venezuela.

To search for overdensities our available tool was the QUEST collaboration survey data. This survey uses a 1 meter telescope located in Merida, Venezuela. The telescope has a camera with a 16 CCD array performing drif scanning mode in 4 filters and two declination bands (centered at -1 and $-3 \mathrm{deg}$ ). The survey covers a large range in right ascension (RA) and goes repeated times over the same part of the sky (on average 35 times each piece), allowing for a variability survey to derive from it. 


\section{The VSS}

The QUEST survey generated a catalog of about 650 photometricaly selected stars. Providing estimates for their periods, times of maximuum light and distances. It also provided an estimate of the expected number of type ab RR Lyrae (RRLy) stars at a particular distance from the galactic center.

Figure 1 is a radial plot displaying RA on the angular coordinate and V magnitude on the radial coordinate. $\mathrm{V}$ magnitude is in the case of RRLy a proxy for distance. All RRLy stars in both declination bands, represented by stars and triangles, are plotted. The two most prominent groups are one identified as debris from Sagittarius, at about RA of 15 hours and V mag of about 19, and another called the $12.4 \mathrm{hr}$ clump at about 12.4 hours in RA and V mag of around 17. This work concentrates on our findings as we studied the $12.4 \mathrm{hr}$ clump.

The key to this study is this very strong and firm first step towards the identification of possible stream candidates, the use of standard candles as tracers of substructure.

The discovery of a moving group whithin the clump was performed by analysing a combination of Blue Horizontal Branch stars (BHBs) and RRLy stars targeted on the densest part of the clump as seen by QUEST. Although small the initial sample (Duffau et al. 2006) was large enough to allow for statistical analysis by means of the Shapiro-Wilk test applied to the radial velocity histogram of the sample. The test revelead that the central sample at the position of the densest location in the clump was significantly different from the expected gaussian distribution of a non-rotating halo model. The difference being caused primeraly by a sharp velocity peak at large positive velocities. Extracting from the sample ( containing 10 BHBs and 18 RRLy stars) the likely VSS member stars, we obtained a mean velocity of $126 \mathrm{~km} / \mathrm{s}$ and a metallicity of -1.84 from the RRLy available data. Observing that the metallicity spread was larger than the estimated errors a first suggestion was made as to the nature of the VSS, identifying it as possible debris from an accreted small galaxy more likely than from a classical GC (single stellar population).

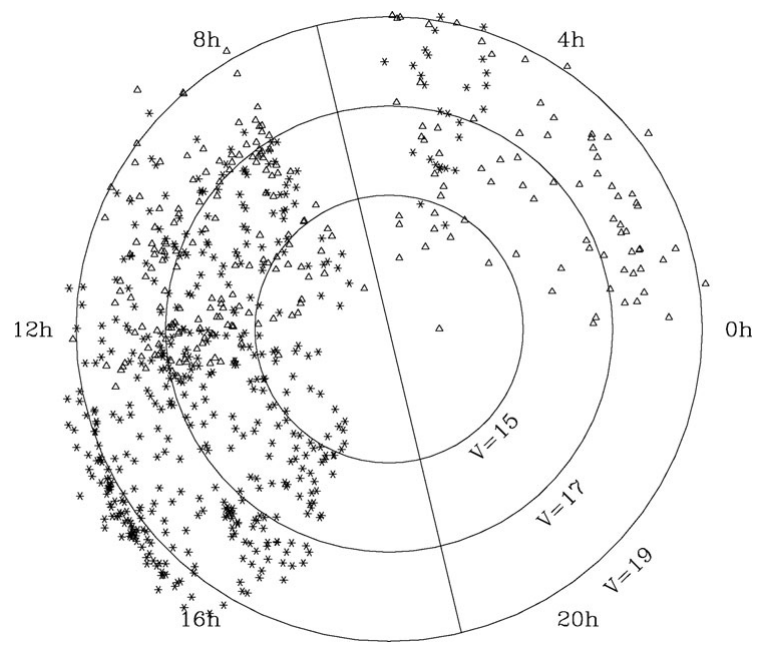

Figure 1. Distribution of photmetrically selected RRLy stars from QUEST. Stars come from declination band at -1 degrees and triangles, from declination band at -3 degrees. 


\section{The extended sample}

Opening up the sample to smaller distances, we observed 51 RRLy stars at similar RA as originally done but at distances between 13 and $21 \mathrm{kpc}$ from the Sun. This encompasses approximately the region of the $12.4 \mathrm{hr}$ clump. Most of the stars have both metallicity and radial velocity estimates.

On a parallel project by the QUEST collaboration (Vivas et al. 2008), another 30 RRLy stars were observed on the same direction but at distances between 4 and $12 \mathrm{kpc}$. Both samples were combined into one large sample to study the extent of the VSS in distance.

Slicing of the sample in radial distance sectors for analysis, we defined five groups, below $7 \mathrm{kpc}$, from 7 to $10 \mathrm{kpc}$, from 10 to $13 \mathrm{kpc}$, from 13 to $18 \mathrm{kpc}$ and finally from 18 to $23 \mathrm{kpc}$. The original VSS was found on the more distant group. Applying the Shapiro-Wilk test we determined that 4 out of the 5 resulting velocity histograms are not normally distributed, suggesting the presence of substructure. Using both the spatial and radial velocity information available we applied a modified form of the stellar pair search (SPS) technique to the identification of the main features in this radial velocitydistance from the Sun plane. The SPS technique developed by Clewley and Kinman in 2006 (Clewley, L. \& Kinman, T. D. 2006), searchs for neighboors in the 4 dimensional space defined by the three spatial directions and the radial velocity. Considering the typical errors involved, it connects pairs of stars that fullfill a criterion of closeness in this 4 dimensional space. The significance of the groupings is found by comparing, by means of montecarlo realisations, the likelyhood of finding such groupings as random occurances on a non-rotating RRLy model of the halo.

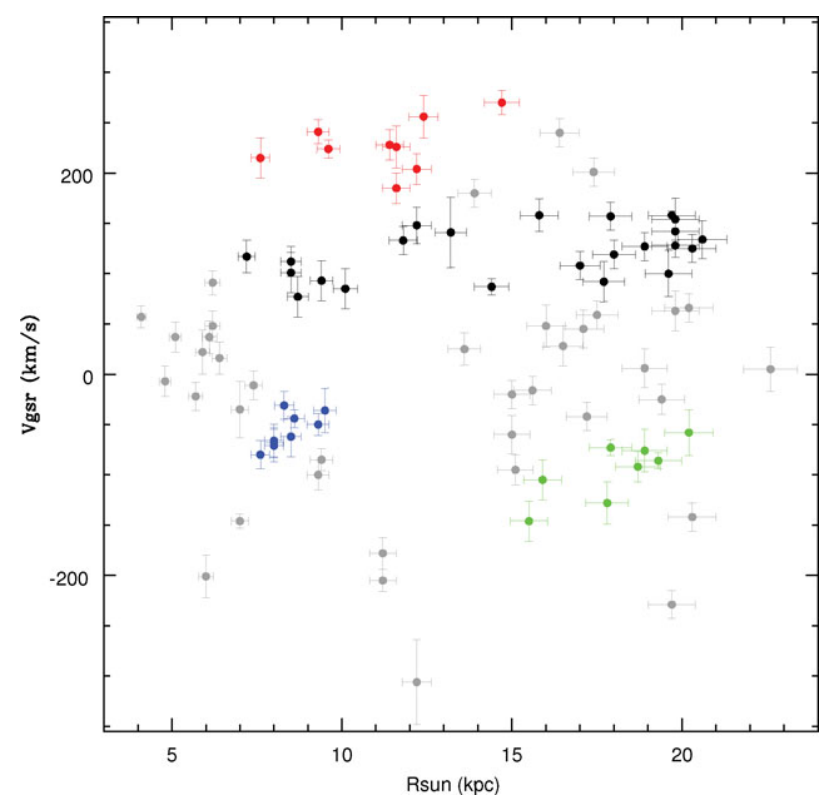

Figure 2. The four most significant groups found using the SPS technique are displayed in this distance from the Sun - radial velocity on the Galactic Standard of Rest plane. The black dots represent the stars associated with the VSS. Red, blue and green dots display the location of the other less relevant groups likely associated with different substructures. 


\section{Results}

Displayed in Figure 2 is the result of using SPS on our data. Four are the most statistically significant groups with less than a 2 percent chance to be the product of fluctuations over a smooth halo model. The most important of which (black dots) contains the original VSS candidates and extends the sample to even smaller radii outside the area of original clump. This group has 23 members and preserves the large metallicity spread originally found for the VSS. The mean radial velocity is now $129 \mathrm{~km} / \mathrm{s}$ and the probability of finding such a group as a result of fluctuations over a smooth halo model is only 0.08 percent. This does not mean that the rest of the stars are not related to this or other groups in this plane, but states the location of the most prominent part of the moving group.

In the area where the VSS was found, several other groups have found stars with similar velocities or overdensities at similar distances as the VSS. Every new group adding a piece of information to the puzzle. Large areas have been identified as containing overdensities as the Virgo Overdensity (VOD) by Juric et al. (2008), at distances consistent with the VSS location, and others have found different groups of stars outside the QUEST observing region with velocities similar to those of the VSS, like Newbergs S297+63-20.5 (Newberg et al. 2002).

In summary a stream of stars at high positive radial velocities seems to be mixed with a large extended overdense region. Dana Casetti-Dinescu and collaborators (CasettiDinescu, D. et al. 2009) have identified one RRLy star from the Quest catalog with velocity measurements from SEGUE II and applied the QUEST methods to obtained the mean velocity and find it to be a likely member of the VSS. The orbit determined from the proper motion of this star is shown in red and for comparison the orbit of Sgr is shown in green. From the GCs located in the path of the orbit one likely association has been drawned with NGC 2419. The most important idea to take away from this work is that the estimated orbit is an eccentric one with the VSS located near a turning point.

When orbits like that are followed on models of the disruption of satellites the result often shows the presence of shells and large accumulations of stars on the vicinity of the turning points, as the figure shows, possibly explaining the presence of a stream and large overdensity at similar positions. The VSS and related subtructures could be the result of the accretion of a satellite in such an eccentric orbit.

\section{References}

Casetti-Dinescu, D., Girard, T., Majewski, S., Vivas, A. K., \& 5 other authors 2009, ApJ 701, 29

Clewley, L. \& Kinman, T. D. 2006, MNRAS, 371, 11

Duffau, S., Zinn, R., Vivas, A. K., Carraro, G., Mendez, R. A., Winnick, R., \& Gallart, C. 2006, ApJ (Letters), 636, L97

Juric, M., Ivezic, Z., Brooks, A., Lupton, R., \& 23 other authors 2008, ApJ 673, 864

Martinez-Delgado, D., Penarrubia, J., Juric, M., \& Alfaro, E. J. 2007, ASPC 374, 195

Newberg, H., Yanny, B., Rockosi, C., Grebel, \& 15 other authors 2002, ApJ 569, 245

Vivas, A. K., Zinn, R., Andrews, P., Bailyn, C., \& 32 other authors. 2001, ApJ (Letters), 554, 33

Vivas, A. K., Jaffe, Y. L., Zinn, R., Winnick, R.,Duffau, S., \& Mateu, C. 2008, AJ, 136, 1645 